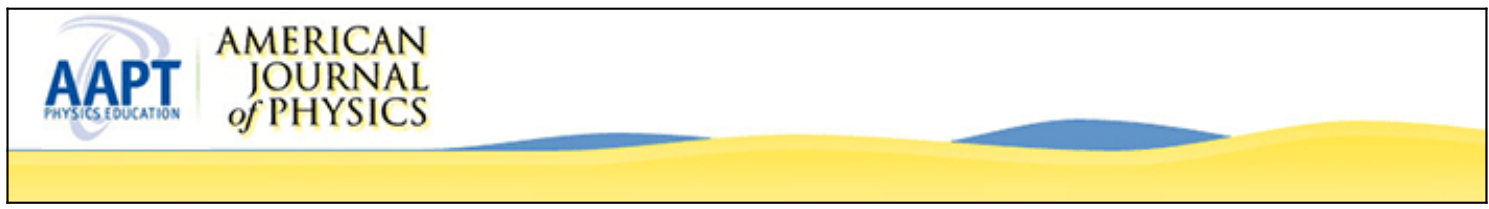

\title{
Crystals and X-Rays
}

Kathleen Lonsdale and Jesse W. M. DuMond

Citation: American Journal of Physics 18, 325 (1950); doi: 10.1119/1.1932583

View online: http://dx.doi.org/10.1119/1.1932583

View Table of Contents: http://scitation.aip.org/content/aapt/journal/ajp/18/5?ver=pdfcov

Published by the American Association of Physics Teachers

\section{Articles you may be interested in}

Imaging $x$-ray crystal spectrometers for KSTAR

Rev. Sci. Instrum. 74, 1997 (2003); 10.1063/1.1535243

Compact convex crystal xray spectrometer

Rev. Sci. Instrum. 62, 2501 (1991); 10.1063/1.1142225

The Twisted Crystal XRay Spectrometer

Rev. Sci. Instrum. 40, 732 (1969); 10.1063/1.1684054

Versatile Double Crystal XRay Goniometer

Rev. Sci. Instrum. 39, 1434 (1968); 10.1063/1.1683126

THE MULTIPLE CRYSTAL XRAY SPECTROGRAPH

Rev. Sci. Instrum. 1, 88 (1930); 10.1063/1.1748677

\section{WebAssign}

\section{Free Physics Videos}

Add these videos and many more resources - free with WebAssign. 
The estimated standard error of $b^{\prime}$ is

$$
s\left(b^{\prime}\right)=(2 m)^{\sharp}\left\{1 /\left(\Sigma_{m} x_{i}-\Sigma_{m} x_{i}\right)\right\} s^{\prime},
$$

and the estimated standard error of the fitted value $y(x)$ is

$$
s(y)=\left\{\left(s^{\prime 2} / n\right)+(x-\bar{x})^{2} s^{2}\left(b^{\prime}\right)\right\}^{3} .
$$

If $r^{\prime}=0.6745 s^{\prime}$ is substituted for $s^{\prime}$ in the above formulas, the corresponding probable errors are obtained. In addition, $r$ may be obtained by means of Peters' formula.

P. G. GUEST

Universily of Sydney

Sydney, Australia

1 P. F. Gaehr, A m. J. Physics 15, 430 (1947); 16, 359 (1948).

2 C. D, Cooksey, Am. J. Physics 16, 189 (1948).
3 Jeffreys, Theory of probability (Oxford, ed. 2), p. 193.

\section{ANNOUNCEMENTS AND NEWS}

\section{Book Reviews}

Crystals and X-Rays. Kathleen Lonsdale. Pp. 199, $8 \frac{1}{2} \times 5 \frac{1}{2}$ in. D. Van Nostrand Company, Inc., New York, 1949. Price $\$ 3.75$.

This justly celebrated research authority has written a small and very compact book of remarkable value to students of physics and chemistry alike. The variety and scope of the intensely interesting material covered and the clarity, directness, and vividly imaginative quality of its expository style set it apart as a unique and remarkable performance. The book is definitely not a popularization written for the lay public. As the author makes clear in her foreword, her aim is to introduce a complex and beautiful domain of science in a compact and therefore rapid way to science students wholly or partially unfamiliar with it. It is not a treatise nor a textbook but in the author's own words is intended to persuade the reader "to pass on from this book to textbooks which are much more thorough, precise and specialized." Quoting her again, "Many people who know a great deal about chemistry and physics and other subjects, however, do not fully realize the value of $x$-ray crystallography as a tool; not merely an industrial tool, but a tool by means of which other sciences may be better understood."

This reviewer has read every word in this book with intense interest and admiration. For more than twentyfive years, he has used x-rays extensively as a research tool to answer questions in atomic and nuclear physics and has had much to do with many of the topics covered by Kathleen Lonsdale's book (although he has never engaged in $\mathrm{x}$-ray crystallography for its own sake). Crystals and $X$-Rays has revealed to him many new and valuable facets of this astonishingly beautiful and fruitful subject which he would otherwise have overlooked, and he feels sure that many another physicist of maturity could profit equally by reading it.

Many of us can readily recall the days when chemists and physicists felt it was unsafe to think (much less talk) of atoms, electrons, and nuclei as real entities. They were abstractions or mental concepts elaborated by the mind out of the raw material of reality. The real raw material consisted of "pointer readings" or "space-time coincidences" and atoms or electrons were abstractions to help us interpret this raw material. They still are abstractions, of course, but such extreme logical caution is no longer a fetish emphasized by scientists. There used to be a joke about the philosopher who called a physicist's attention to a shorn sheep and who was sternly reprimanded by the latter with the reply "yes, I see a sheep shorn on this side." Today this story might hardly be understood. In reading Crystals and $X$-Rays perhaps the most striking single impression this reviewer has received is the tremendous part that the research results described therein have played in this change of attitude as regards the reality of the atomic world; a world which has never been directly seen at all and yet for which the evidence is now so overwhelming that it stands almost on the same level of reality as the familiar objects of everyday life. Along with the Wilson cloud chamber, the Geiger counter, Aston's mass spectrograph, Stern's and Rabi's molecular beams and the revelations of high frequency spectra, one must accord the field of $x$-rays a very prominent place in this revolution.

Mrs. Lonsdale paints this fascinating picture with broad, uninhibited and vivid brush strokes, her one aim being, at every point, maximum clarity and conciseness. To quote a single example of this style, she says, "An investigation of the thermal expansion of the phthalocyanines, plate-like organic molecules which stack themselves together in the crystal in a zigzag way, has shown that the expansion is not only anistropic, but may even be negative in certain crystal directions, simply because the vibrating molecules can pack themselves more comfortably if they turn slightly, instead of merely taking up more room in all directions." The reader, like Alice, is thus led so easily and by such homely common sense analogies into this wonderland of atomic reality that he scarcely realizes what has happened to him-that imagination and reason combined with the scientific experimental method have permitted him to shrink, at least with the eyes of the mind, until he lives with and sees the atoms in their lattice work patterns vibrating as the waves of thermal motion ripple and cross over the atomic planes. More than half of the book is devoted to explaining with great clarity the methods used in the analysis of crystal structure and one receives a just impression of the tremendous amount of labor and ingenuity this subject involves, particularly as regards the reduction and interpretation of the data. There is a wealth of fine illustrations, both line drawings and half-tone reproductions, of diffraction patterns, Laue and powder 
diagrams, rotating crystal spectra and the like, which help enormously to easy comprehension.

The style is not only lucid but witty. There are occasional and delightful flashes which reward the reader with a hearty laugh at the most unexpected places but I shall not spoil the enjoyment by quoting them here.

One serious criticism that, in this reviewer's opinion, can justly be aimed at this work, is the paucity of references to the original literature. All that is given is a list of books, uncorrelated with the text, covering a page and a half at the end. A few of the illustrations have accompanying references. Frequently, intensely interesting material is necessarily compressed into a single sentence and the reader is disappointed to find little or no clue to help him read further about it in journal articles or books. This is in complete disaccord with the stated object of the book. The author should not hesitate to introduce numbered footnote references to the original literature on every important topic she mentions. This would make the book worth its weight in gold. If she suffers from an aversion (not shared by this reviewer) to cluttering the foot of every page in this way, she could follow the excellent example of M. Siegbahn and place all the references at the end in order of date.

This reviewer has noticed a few minor slips. On p. 24, the numerical constant $(h c / e)$ in the equation $\lambda_{\min } V_{\max }$ $=1.234 \times 10^{-4}$ is now believed to have a value much nearer $1.239 \times 10^{-4}$.

An old and sturdy but indefensible misconception which seems very widespread among $x$-ray crystallographic people is repeated on p. 140 in the discussion of primary extinction. The reviewer believes this has taken root from the unfortunate practice of calling the quantity $\rho=E \omega / I$ a reflection coefficient when in fact it is an angle, a measure of the angular range over which Bragg reflection occurs. (The name "integrated reflection coefficient" seems more appropriate for this quantity.) In explaining how the reflections from a nearly ideally perfect crystal come to be much weaker than those from a more imperfect one, the author gives a correct and vivid picture of the strong interference effects from the multiple to-and-fro reflections between planes which rapidly extinguish the amplitude in the primary direction because of the change of phase of $\pi / 2$ at each reflection and hence $\pi$ after two or an even number of successive to-and-fro reflections "so that a twice reflected beam is opposite in phase to the incident primary beam and partially interferes with it. Both reflected and transmitted beams, in fact, are frittered away, as it were, by interference; so that after passing through some $10^{4}$ crystal planes there is no primary beam left in the right direction for Bragg reflection, and the reflected beam is reduced not only by interference, but by the fact that the rest of the crystal, under the skin, cannot reflect a primary beam that does not reach it." [Quoted from p. 140.]

Now this reviewer believes the quoted statements are nonsense but he is also well aware that Mrs. Lonsdale here sins in very good company.

Studies with the two-crystal spectrometer have pretty well verified the correctness of the dynamical theory of $\mathrm{x}$-ray reflection in perfect crystal lattices, first so ably worked out by $C$. G. Darwin. If we consider the ideal case of a highly monochromatic beam of, say, 0.7A x-ray incident on a "perfect" crystal at an angle defined with much sharper definition than the width of the diffraction pattern, then at the most favorable angle the crystal can reflect nearly 100 percent of the beam. In this condition, the reason why the beam does not penetrate deeply into the crystal is because the interference effects described make the reflection so efficient, not because interference "fritters away" any intensity. (The interference of coherent wavelets cannot reduce the total energy in these wavelets! It merely redistributes this energy, extinguishing it in certain regions or directions and piling it up in others.) Only absorption processes which degrade the $\mathrm{x}$-ray energy can fritter away the incident or reflected beams and precisely in the case of strong primary extinction in nearly ideal lattices absorption is reduced to a minimum because of the slight penetration. Thus, "frittering away" is here an incorrect term. If we disturb the surface mechanically or otherwise reduce the approach to ideal perfection of the lattice, the reflection (integrated over its entire angular range) is enhanced not by an increase in the peak reflection coefficient (which cannot exceed 100 percent!) but by reason of the increased angular range coming from the disorientation. Thus, it is misleading to say that "primary extinction gives weak reflections." A preferable statement would be that the approach to a very ideal lattice is accompanied with strong primary extinction and also results in Bragg reflections limited to very narrow angular ranges which place a severe limitation on the solid angle into which each excited atom in the $\mathrm{x}$-ray tube target can radiate. Usually slit spectrometers of relatively low angular resolving power are used. Since the angular widths over which Bragg reflection occurs in very perfect crystals are of the order of a second or so of arc, the angle, and hence the power associated with the reflection, can easily be increased many factors of ten without any detectable change in the apparent width of the reflection unless really refined instruments such as the two-crystal spectrometer are used in the study. One cannot help wondering if it is not significant that there is nowhere any mention in Mrs. Lonsdale's book of the two-crystal instrument.

Another surprising omission is the almost complete absence of any discussion of the Compton effect and of modified or incoherent scattering. In the reviewer's opinion this, bearing as it does directly on the particle-wave duality, is by far the most far-reaching and significant thing that has come out of $\mathrm{x}$-ray research. However, in fairness we must recall that Mrs. Lonsdale has put the word "crystals" first in her title.

The reviewer is entirely in sympathy with the general conciseness and brevity which makes the book so suitable to its stated purposes. Nevertheless, he feels that at a few points one or two extra clarifying sentences would have been real improvements. A perhaps trivial example is on p. 13 in Fig. 5 and the accompanying discussion in which one learns that "a divergent incident beam gives a convergent diffracted beam" and it is stated that this "could be quite simply explained if the slightly divergent primary 
beam was being reflected from some kind of planes in the crystal," The neophyte would be considerably confused by these words and by the picture for he would recall that the laws of image formation and of reflection by plane mirrors call for a divergent reflected beam if the incident beam is divergent. It would have greatly clarified this to have added a word of explanation in a footnote to the effect that the planes are frequently distorted so that their directions are somewhat ill-defined and that the dominant influence here is not the specular reflection law (requiring equal glancing angles of incidence and reflection with the plane) but the Bragg law coming from the periodicity normal to the planes which imposes still more imperatively that the angle of deviation of the beam shall be constant.

Again on p. 32 the well-known modified equation

$$
n \lambda_{0}=2 d\left(1-\delta / \sin ^{2} \theta\right) \sin \theta,
$$

containing the factor correcting for the effect of refraction is stated without pointing out that in this form it is valid for only one special case, that of Bragg reflection by planes parallel to the boundary surface of the crystal which must also be the common surface of entry and of exit of the beam. This equation as it stands is quite inapplicable to the more general cases of Laue reflection.

It would be wrong to fail to point out the above criticisms but the reviewer realizes that, by so doing, these very minor blemishes on an outstandingly excellent work are unduly magnified. They are, as a matter of fact, fairly typical errors in works on $\mathrm{x}$-ray crystallography that have been propagated now for many years from book to book and should be corrected. Mrs. Lonsdale's work is so outstandingly excellent in most respects that their repetition can surely be forgiven.

$$
\begin{aligned}
& \text { JEsse W. M. DuMOND } \\
& \text { California Institute of Technology }
\end{aligned}
$$

Albert Einstein: His Work and Its Influence On Our World. Leopold Infeld. Pp. 125. Charles Scribner's Sons, New York, 1950. Price $\$ 2.00$.

In this modest volume Professor Infeld gives a popular account of relativity theory, and of the other scientific achievements of Albert Einstein. By virtue of his own contributions to relativity theory, his associations with Einstein, and his past literary work, the author is well qualified for this task. The story he tells is one, to be sure, that by now has been told many times, and on many different levels of scientific sophistication; but the permanence and depth of Einstein's work and the appeal of his devoted life are valid themes that bear much retelling and rereading.

After some introductory remarks the author arranges his book into four chapters: The First Einstein Revolution gives the development of the special theory of relativity; The Second Einstein Revolution is concerned with the general theory and its applications in cosmology; in The Unfinished Revolution Einstein's work in the quantum theory of light and in Brownian motion is discussed; and the final chapter, Beyond the Revolutions, is devoted to attempts toward a unified field theory and to other than scientific activities of Einstein. The treatment is every- where nonmathematical, and no previous knowledge of physics is assumed; but, as seems to be characteristic of many of the best popular science expositions, previous scientific knowledge does help the reader. Only the thoroughly versed relativity theorist, I should think, would be bored with Professor Infeld's discussions of the theory.

There is one particular in which the author's presentation, it seems to me, could be misleading. In discussing the Lorentz transformation he uses Fifth Avenue as one coordinate system, and a moving bus on the avenue as another. The bus driver finds his clock to have a slower rhythm, because he is in a moving system, than do the clocks along Fif th Avenue. The paradox of the twins is then introduced, a twin in the bus being taken as the one becoming younger than his brother. The reader may be confused at this point, since the equivalence of coordinate systems in relative motion to each other has been stressed, and yet in the illustration the Fifth Avenue coordinate system is taken as an "absolute" one by which the bus driver determines his motion. Actually we might expect that the bus driver would find his clock to be normal, but the "moving" Fif th Avenue clocks to decrease in tempo.

The title of this volume refers to Einstein's influence as well as to his work. Professor Infeld does give some indication of the many effects that have come from Einstein's work, but he gives no systematic treatment, in the manner of a cultural historian or critic. (The reader interested in Einstein's influence, scientific and philosophical, can do no better than look to the remarkable volume, Einstein: Philosopher-Scientist, recently published in the Library of Living Philosophers series.) However, the author's personal familiarity with Einstein gives the book a warm touch that recommends it. In communicating to the reader something of the personality of Einstein, Professor Infeld also gives us some feeling of how fortunate all scientists are that Einstein is today to the generality of mankind the symbol of science.

\section{RICHARD SCHLEGEL} Michigan State College

Science and Civilization. Robert C. Stauffer, Editor. Pp. $212+x i i i, 5 \frac{1}{2} \times 8 \frac{1}{2}$ in. University of Wisconsin Press, Madison, 1949. Price $\$ 2.50$.

This volume is a compilation of eight addresses delivered at a symposium in 1949 under the sponsorship of the Wisconsin History of Science Group, in celebration of the One Hundredth Anniversary of the founding of the University of Wisconsin.

The titles of these addresses and their authors are listed in order, with brief comments.

Aristotle and the Origins of Science in the West. RICHARD P. McKeon.-In this very learned discussion of the philosophy of Aristotle, the author concludes that the contribution of Aristotle to the origins of science in the West must be stated on three levels:

A. Aristotle discovered facts and constructed theories that had profound influence on later scientists.

B. His terms and ideas, his deductions and theories 